\title{
Correspondence
}

Psychological Medicine, 46 (2016).

doi:10.1017/S0033291716000623

First published online 16 May 2016

\section{Comparison of cognitive functions, pre-morbid conditions and clinical characteristics between brief psychotic disorder and schizophrenia}

Brief psychotic disorder (BPD) and acute and transient psychotic disorder (ATPD) are two related but different concepts used to define psychotic disorders with acute onset and early remission by the Diagnostic and Statistical Manual of Mental Disorders, fifth edition (DSM-5) and the International Classification of Diseases, tenth edition (ICD-10), correspondingly (Gaebel \& Reed, 2012). ATPD is a broad category which integrated various concepts including bouffée délirante (Pichot, 1986), cycloid psychosis (Perris, 1986) and the reactive and schizophreniform psychoses (Stromgren, 1986), while BPD is a diagnosis with duration of symptoms of 1 month or less and eventual full return to pre-morbid functioning. Studies in ATPD suggest that patients with ATPD are a higher proportion of females, more likely to have stressful life events before onset of illness and full recovery (Pillmann, et al. 2012; Rusaka \& Rancāns, 2014; Castagnini et al. 2016). Research in ATPD has grown in recent years, but many of the studies were retrospective in nature and of inpatient records, and there have been only a few studies in BPD (Pillmann et al. 2002a, b). There was only moderate concordance between BPD and the polymorphic subtype (F23.0) of ATPD, while the schizophrenia-like subtype (F23.2) of ATPD was in concordance with schizophreniform disorder (Pillmann et al. 2002a) and little is known about the cognitive functions and premorbid condition in this population. The present study was designed to compare the pre-morbid condition, cognitive function, and demographic and clinical characteristics between patients with BPD and patients with schizophrenia in a prospective study with an outpatient sample.

A total of 42 patients with BPD (16 men and 26 women, mean age 36.02 years) and 157 patients with schizophrenia (75 men and 82 women, mean age 37.61 years) out of 360 patients were consecutively recruited between June 2009 and August 2011 from a population-based territory-wide study of early psychosis in Hong Kong targeting adult-onset first-episode patients [the Jockey Club Early Psychosis (JCEP)
Project; Hui et al. 2014, 2015]. Written informed consent was obtained from all patients. The study was approved by the Institutional Review Boards and conducted in accordance with Good Clinical Practice and the Declaration of Helsinki.

Basic demographic information including age, gender, years of education and marital status were recorded. Diagnosis was made according to DSM-IV criteria and reconfirmed at 6 months following the first episode by two experienced psychiatrists based on a best-estimate consensus using all available information, including the validated Chinese version of the Structured Clinical Interview for DSM-IV (So et al. 2003), medical records, history from informants, and case workers of the JCEP Project. Age of onset, presence of life events in the prior 6 months, psychiatric hospitalization at entry and family history of mental illness were evaluated.

Pre-morbid functioning during childhood, adolescence and adulthood were evaluated using the Premorbid Adjustment Scale (PAS; Cannon-Spoor et al. 1982). Pre-morbid schizoid and schizotypal traits were assessed by the assessment of Premorbid Schizoid and Schizotypal Traits (PSST; Foerster et al. 1991). Positive and negative symptoms were assessed using the Positive and Negative Syndrome Scale (PANSS; Kay et al. 1987). Cognitive functions were assessed using a comprehensive battery of neurocognitive tests, which included digit span (forward and backward), visual patterns test, logical memory (immediate and delay recall), verbal fluency test and digit symbol substitution test. Functioning level was assessed using the Social Occupational Functioning Assessment Scale (SOFAS; Goldman et al. 1992).

All statistical analyses were performed using IBM SPSS version 23.0 (IBM Corp., USA). Differences in the basic demographic, clinical, functional and neurocognitive characteristics were determined using independent $t$ tests for parametric continuous variables, and $\chi^{2}$ tests for categorical variables. The level of statistical significance for all analyses was set at $p<0.05$.

There were $35.7 \%(n=15)$ of patients with BPD and $24.8 \%(n=39)$ of patients with schizophrenia who were married. Family history of mental illness was present in $31.0 \%(n=13)$ of patients with BPD and $29.3 \%$ $(n=46)$ of patients with schizophrenia. A life event was present in $47.6 \%(n=20)$ of patients with BPD and $48.4 \%(n=76)$ of patients with schizophrenia. All of these were not statistically different between the two groups. The other demographic and clinical characteristics are listed in Table 1. 
Table 1. Clinical characteristics

\begin{tabular}{lcrr}
\hline Baseline characteristics & Brief psychotic disorder $(n=42)$ & Schizophrenia $(n=157)$ & $t$ \\
\hline Age, years & $36.02(7.89)$ & $37.61(7.92)$ & 1.155 \\
Education, years & $11.40(3.60)$ & $10.73(3.55)$ & -1.095 \\
Age at onset, years & $35.76(7.95)$ & $35.36(8.52)$ & -0.278 \\
Mean PSST score & $1.06(0.11)$ & $1.18(0.26)$ & 0.275 \\
Mean PAS score & $0.11(0.13)$ & $0.18(0.17)$ & 0.782 \\
PANSS total score & $41.14(10.01)$ & $47.40(13.13)$ & 2.519 \\
Digit span forward & $11.71(2.29)$ & $11.16(2.40)$ & 0.000 \\
Digit span backward & $5.95(2.85)$ & $6.32(2.96)$ & 0.013 \\
Visual patterns test & $7.23(2.28)$ & $7.31(2.17)$ & -1.310 \\
Logical memory test & & & 0.715 \\
$\quad$ Immediate recall & $9.20(4.73)$ & $8.18(4.50)$ & -0.143 \\
$\quad$ Delay recall & $6.40(4.54)$ & $6.49(4.61)$ & 0.192 \\
Verbal fluency test & $16.64(4.50)$ & $15.46(5.65)$ & -1.258 \\
Digit symbol substitution test & $67.18(25.64)$ & $59.67(18.11)$ & 0.105 \\
SOFAS score & $64.74(14.31)$ & $57.06(12.16)$ & -1.254 \\
& & & 0.210 \\
\hline
\end{tabular}

Data are given as mean (standard deviation).

PSST, Premorbid Schizoid and Schizotypal Traits; PAS, Premorbid Adjustment Scale; PANSS, Positive and Negative Syndrome Scale; SOFAS, Social and Occupational Functioning Assessment Scale.

The mean PSST score, mean PAS score and PANSS total score were lower in patients with BPD than patients with schizophrenia. Patients with BPD had a significantly higher SOFAS score and a higher proportion of psychiatric admission than patients with schizophrenia $\left(81.0 \%\right.$ v. $\left.55.4 \% ; \chi^{2}=9.068, p=0.003\right)$.

To the best of our knowledge, this is the first study to examine the cognitive functions and pre-morbid conditions in patients with BPD. We found that patients with BPD are different from patients with schizophrenia prior to onset of illness with fewer schizoid and schizotypal traits and better pre-morbid functioning. This is in consistent with the concept that BPD is a psychotic disorder which has an acute onset in contrast to schizophrenia which has personality predisposition and functional decline in the prodromal period. Patients with BPD also had a higher proportion of psychiatric admission than patients with schizophrenia. This may be explained by the acute change in condition which the carer and health care professionals found difficult to manage in the community. Although patients with BPD had milder severity of psychopathology, and better social and occupational functioning than patients with schizophrenia, a considerable degree of residual symptoms was still observed even after stabilization of the first episode of psychotic illness. Furthermore, we did not find any difference in cognitive functions including attention, memory and executive function between patients with BPD and patients with schizophrenia. The lack of difference may suggest that patients with BPD may not have recovered faster than patients with schizophrenia in terms of cognitive functions after a psychotic episode. Our previous finding in first-episode psychosis patients suggests that there may be a time lag between improvement in cognitive functions and symptoms (Hui et al. 2012). These findings warrant future studies to examine if patients with BPD can achieve full remission after a psychotic episode, especially for a subgroup of BPD with repeated brief psychotic episodes (Pillmann et al. 2002b). In contrast to some previous findings in ATPD, we found no significant difference in gender ratio, marital status, family history of mental illness and presence of life events between patients with BPD and schizophrenia (Castagnini \& Berrios, 2009). This suggests that findings in ATPD may not be applied directly to BPD and more studies in BPD are needed.

Our study suggests that BPD is different from schizophrenia in terms of patients' pre-morbid conditions, severity of psychopathology and social functioning. Future studies using a longitudinal design examining the change in cognitive functions in comparison with normal controls and its importance in prediction of relapse and diagnostic transition may help to identify a subgroup of patients for more intensive care. Major change in the subtyping of ATPD has been proposed in the coming ICD-11; we hope that more research in BPD can help to understand the characteristics of acute psychotic disorders to facilitate future refinement of the classification system. 


\section{Acknowledgements}

This work was supported by funding from the Hong Kong Jockey Club Charities Trust (grant number 21009144).

\section{Declaration of Interest}

E.Y.H.C. has served on the advisory board for Otsuka; and has received research funding from AstraZeneca, Janssen-Cilag, Pfizer, Eli Lilly, Sanofi-Aventis and Otsuka, and an educational grant from Janssen-Cilag. The other authors declare no conflict of interest in this study.

\section{References}

Cannon-Spoor HE, Potkin SG, Wyatt RJ (1982). Measurement of premorbid adjustment in chronic schizophrenia. Schizophrenia Bulletin 8, 470-484.

Castagnini A, Berrios GE (2009). Acute and transient psychotic disorders (ICD-10 F23): a review from a European perspective. European Archives of Psychiatry and Clinical Neuroscience 259, 433-443.

Castagnini AC, Munk-Jørgensen P, Bertelsen A (2016). Short-term course and outcome of acute and transient psychotic disorders: differences from other types of psychosis with acute onset. International Journal of Social Psychiatry 62, 51-56.

Foerster A, Lewis S, Owen M, Murray R (1991). Pre-morbid adjustment and personality in psychosis. Effects of sex and diagnosis. British Journal of Psychiatry 158, 171-176.

Gaebel W, Reed GM (2012). Status of psychotic disorders in ICD-11. Schizophrenia Bulletin 38, 895-898.

Goldman HH, Skodol AE, Lave TR (1992). Revising Axis V for DSM-IV: a review of measures of social functioning. American Journal of Psychiatry 149, 1148-1156.

Hui CL, Chang WC, Chan SK, Lee EH, Tam WW, Lai DC, Wong GH, Tang JY, Li FW, Leung KF, McGhee SM, Sham PC, Chen EY (2014). Early intervention and evaluation for adult-onset psychosis: the JCEP study rationale and design. Early Intervention in Psychiatry 8, 261-268.

Hui CL, Lau WW, Leung CM, Chang WC, Tang JY, Wong GH, Chan SK, Lee EH, Chen EY (2015). Clinical and social correlates of duration of untreated psychosis among adult-onset psychosis in Hong Kong Chinese: the JCEP study. Early Intervention in Psychiatry 9, 118-125.

Hui CL, Longenecker J, Wong GH, Tang JY, Chang WC, Chan SK, Lee EH, Dunn EL, Miao MY, Yeung WS, Wong CK, Chan WF, Tang WN, Chen EY (2012). Longitudinal changes in semantic categorization performance after symptomatic remission from first-episode psychosis: a 3-year follow-up study. Schizophrenia Research 137, 118-123.

Kay SR, Fiszbein A, Opler LA (1987). The Positive and Negative Syndrome Scale (PANSS) for schizophrenia. Schizophrenia Bulletin 13, 261-276.

Perris C (1986). The case for the independence of cycloid psychotic disorder from the schizoaffective disorders. In Schizoaffective Psychoses (ed. A. Marneros and M. T. Tsuang), pp. 272-308. Springer: Berlin.

Pichot P (1986). The concept of 'bouffée délirante' with special reference to the Scandinavian concept of reactive psychosis. Psychopathology 19, 35-43.

Pillmann F, Haring A, Balzuweit S, Blöink R, Marneros A (2002a). The concordance of ICD-10 acute and transient psychosis and DSM-IV brief psychotic disorder. Psychological Medicine 32, 525-533.

Pillmann F, Haring A, Balzuweit S, Marneros A (2002b). A comparison of DSM-IV brief psychotic disorder with "positive" schizophrenia and healthy controls. Comprehensive Psychiatry 43, 385-392.

Pillmann F, Wustmann T, Marneros A (2012). Acute and transient psychotic disorders versus persistent delusional disorders: a comparative longitudinal study. Psychiatry and Clinical Neurosciences 66, 44-52.

Rusaka M, Rancāns E (2014). A prospective follow-up study of first-episode acute transient psychotic disorder in Latvia. Annals of General Psychiatry 13, 4.

So E, Kam I, Leung CM, Chung D, Liu Z, Fong S (2003). The Chinese-bilingual SCID-I/P project: stage 1 - reliability for mood disorders and schizophrenia. East Asian Archive Psychiatry 13, 7-18.

Stromgren E (1986). Reactive (psychogenic) psychoses and their relations to schizoaffective psychoses. In Schizoaffective Psychoses (ed. A. Marneros and M. T. Tsuang), pp. 260-271. Springer: Berlin.

E. H. M. $\mathrm{LEE}^{1}, \mathrm{C} \cdot \mathrm{L} \cdot \mathrm{M} \cdot \mathrm{HUI}^{1}$, W. C. $\mathrm{CHANG}^{1,2}$

S. K. W. CHAN ${ }^{1,2}$, J. $\operatorname{LIN}^{1}$ AND E. Y. H. CHEN ${ }^{1,2}$

${ }^{1}$ Department of Psychiatry, University of Hong Kong, Hong Kong SAR, People's Republic of China

${ }^{2}$ State Key Laboratory of Brain and Cognitive Sciences, University of Hong Kong, Hong Kong SAR, People's Republic of China

Address for correspondence: C. L. M. Hui, Department of Psychiatry, University of Hong Kong, Queen Mary Hospital, 102 Pokfulam Road, Hong Kong SAR, People's Republic of China. (Email: christy@lmhui.com) 